ROCKY MOUNTAIN

JOURNAL OF MATHEMATICS

Volume 14, Number 4, Fall 1984

\title{
REAL PLACES IN FUNCTION FIELDS
}

\author{
CARLOS ANDRADAS
}

\section{Dedicated to the memory of Gus Efroymson}

In this note we present some results on real places in function fields. Namely we study the existence of real places and specialization chains of real places with given centers, ranks and dimensions. For a complete discussion and details of the proofs we refer to [1]. The results presented here form part of dissertation of the author made under the supervision of Professor D.W. Dubois.

Let $R$ be a real closed field, $K=R\left(x_{1}, \ldots, x_{n}\right)$ a formally real extension of transcendence degree $r$ over $R$ and set $A=R\left[x_{1}, \ldots, x_{n}\right]$. We say that a prime ideal $\mathscr{P}$ of $A$ is locally real if $\mathscr{P}$ is $\left(\beta_{w} \cap A\right)$-convex, where $\beta_{w}$ stands for the partial order of the sums of squares of $K$.

We have the following main result.

THEOREM 1. For each couple of integers $m$, $s$ such that $1 \leqq m \leqq r$, $0 \leqq \operatorname{dim} \mathscr{P} \leqq s$ and $s+m \leqq r$ there exists a real place $\phi$ of $K$ over $R$, centered at $\mathscr{P}$ in $A$, with rank $\phi=m$ and $\operatorname{dim} \phi=s$.

REMARK 2. In particular Theorem 1 includes the existence of real prime divisors centered at a given locally real prime ideal [3].

SKetCh OF THE ProOf. We consider first the case $m=1$ and $\operatorname{dim} \mathscr{P}=0$. Let $V$ be an algebraic model of $K$ with coordinate ring $A$. Then $\mathscr{P}$ corresponds to a central point of $V$ that we assume to be the origin 0 . Now the existence of zero-dimensional rank one real places of $K$ centered at 0 is equivalent to finding formal Zariski-dense curves on $V$ through 0 , i.e., homomorphisms $r: R\left[X_{1}, \ldots, X_{n}\right] \rightarrow R[[t]]$ such that $\operatorname{ker} \gamma=J(V)$ and the order of $\gamma\left(X_{i}\right)$ is positive for all $i=1, \ldots, n$. Therefore the theorem for $m=1, \operatorname{dim} \mathscr{P}=s=0$ follows at once from the following result.

Proposition 3. (Zariski-dense curve selection lemma). Let $S$ be an open semialgebraic subset of the simple points of $V$ such that $0 \in \vec{S}$. Then there exists a formal curve $r$ on $V$ through 0 contained in $S$ and dense in the topology of Zariski in $V$.

The case $m=1, \operatorname{dim} \mathscr{P}=s>0$ is managed by extending the ground 
field $R$. Then to break down the equality $\operatorname{dim} \mathscr{P}=s$ we reduce ourselves to the case of hypersurfaces, and then we blow up the origin adequately, following Zariski's local uniformization theorem. Finally by composing rank one places we reach Theorem 1 for arbitrary $m$ and $s$.

Remark 4. The proof of Proposition 3 is geometric, which forces us to work first over microbial fields (where analytic functions do define "material" curves) and afterwards to extend the result to arbitrary ground fields.

REMARK 5. It is interesting for further applications to point out the following byproduct of the proof of theorem 1 . Set $\triangle=A_{\mathscr{P}} / \mathscr{P} A_{\mathscr{P}}$; assume that $\beta$ is a total order of $K$ such that $\mathscr{P}$ is $(\beta \cap A)$-convex. Then the place $\phi$ of Theorem 1 can be chosen such that its residual field is an ordered extension of $(\triangle, \bar{\beta})$ where $\bar{\beta}=\left(\beta \cap A_{\mathscr{P}}\right) / \mathscr{P} A_{\mathscr{P}}$.

Let us pass to the existence of specialization chains of real places. Let $\mathscr{P}_{m-1} \subset \cdots \subset \mathscr{P}_{1} \subset \mathscr{P}_{0}$ be a chain of prime ideals of $A$ such that there exists a total order $\beta$ in $K$ making each $\mathscr{P}_{i}(\beta \cap A)$-convex. The chain need not be strict. Let $0 \leqq s_{0}<s_{1}<\cdots<s_{m-1}<r$ be integers such that for each $i=0, \ldots, m-1 \operatorname{dim} \mathscr{P}_{i} \leqq s_{i}$ and $s_{i}+m-i \leqq r$. We consider the following problem.

Specialization Problem. Does there exist a specialization chain of real places of $K$ over $R, \phi_{0}, \phi_{1}, \ldots, \phi_{m-1}$, such that for each $i$ the place $\phi_{i}$ : $K \rightarrow \Sigma_{i}, \infty$ is centered at $\mathscr{P}_{i}$ in $A$, rank $\phi_{i}=m-i$ and $\operatorname{dim} \phi_{i}=s_{i}$ ?

We have the following results.

Proposition 6. If $s_{i}=r-m+i, i=1, \ldots, m-1$ (So arbitrary) then the specialization problem has positive answer.

Proof. The hardest situation to handle is when the chain of ideals is not strict but has repetitions. The general technique is induction together with Theorem 1 and Remark 5. In this connection we point out that proposition 6 is false if we fix some of the $\phi_{i}$ 's in advance.

If the chain $\mathscr{P}_{m-1} \subset \cdots \subset \mathscr{P}_{1} \subset \mathscr{P}_{0}$ is strict we say that it has the order-lifting property if for every order $\beta_{i}$ in $A / \mathscr{P}_{i}$ there exists an order $\beta_{i+1}$ in $A / \mathscr{P}_{i+1}$ such that $\beta_{i}=\beta_{i+1} /\left(\mathscr{P}_{i} / \mathscr{P}_{i+1}\right)$. For example, if the chain $\mathscr{P}_{m-1} \subset \cdots \subset \mathscr{P}_{0}$ corresponds to a family of non-singular subvarieties of $V$ then it has the order-lifting property [2].

PROPOSITION 7. If $\mathscr{P}_{m-1} \subset \cdots \subset \mathscr{P}_{0}$ has the order-lifting property and $s_{i}=\operatorname{dim} \mathscr{P}_{i}, i=0, \ldots, m-1$, then the specialization problem has affirmative answer.

Proof. It uses Theorem 1, Remark 5 and Knebusch's real place extension theorem in a process of hunting orders in successive diagrams [4]. 
As a corollary we get

COROLlary 8. Let $V$ be a real variety of dimension 2 or 3. Then any admissible problem of specialization chains of real places of $R(V)$ has affirmative answer.

To finish we conjecture that the specialization chain problem has positive answer in any dimension $r$.

\section{REFERENCES}

1. C. Andradas, Real places in function fields, Dissertation, Univ. of New Mexico, Albuquerque, 1983.

2. G. Brumfiel, Partially ordered rings and semi-algebraic geometry, London Math. Lect. Notes 37, Cambridge Univ. Press, 1979.

3. _- Real valuation rings and ideals. Lect. Notes 959, Springer-Verlag, 1983, 55-97.

4. M. Knebusch, On the extension of real places, Comm. Math. Helv. 47, 1972, 354 369.

Dpto. de Algebra y Fundamentos Fac. de Matemáticas Univ. Complutense MADRID-3 SPAIN. 
